\title{
Caracterização de homicídios e aspectos associados ao uso de drogas ilícitas em uma Capital no Nordeste Brasileiro
}

\section{Characterization of homicide and associated factors with use of drugs in a state capital city in Northeast Brazil}

Alline Oliveira do Nascimento Veloso ${ }^{1}$, Kaio Keomma², Mayrla Sousa Coutinho ${ }^{1}$, Alessandro Leite Cavalcanti ${ }^{1}$

'Programa de Pós-Graduação em Saúde Pública, Universidade Estadual da Paraíba (UEPB) - Campina Grande (PB), Brasil.

${ }^{2}$ Faculdade de Saúde Pública, Universidade de São Paulo (FSP-USP) - São Paulo (SP), Brasil.

DOI: https://dx.doi.org/10.7322/abcshs.v44i3.1203

\begin{abstract}
RESUMO
Introdução: O homicídio é um problema de Saúde Pública no Brasil não raro relacionado com drogas ilícitas. Objetivo: Caracterizar os homicídios e os aspectos associados ao uso de drogas ilícitas. Métodos: Estudo transversal, com abordagem quantitativa, realizado em João Pessoa, no estado da Paraíba, no Brasil, em 2014. Consideraram-se 424 vítimas de homicídios cujos dados foram coletados através de um instrumento próprio, analisados com auxílio de software estatístico, com Teste de QuiQuadrado e Regressão Robusta de Poisson, considerando um nível confiança de 95\% e significância estatística quando $p<0,05$. Resultados: Predominância de vítimas de homicídios homens (93,2\%), não brancos $(97,2 \%)$, com até 30 anos, considerando as faixas etárias de menor de 20 anos (22,6\%), de 20 a 24 (26,7\%) e 25 a 29 (18,8\%), com menos de sete anos de estudo (67,5\%), com histórico de envolvimento com drogas ilícitas (72,5\%) e de encarceramento (59,7\%). Observaram-se associações entre envolvimento com drogas ilícitas e as variáveis: sexo $(p=0,037)$, idade $(p=0,002)$ e histórico de encarceramento $(p<0,001)$. A prevalência ajustada de envolvimento com drogas ilícitas foi $67 \%$ maior entre aqueles com histórico de encarceramento (RP: 1,67; IC95\%: 1,44-1,94) e 28\% menor entre indivíduos com 30 anos ou mais (RP: 0,72; IC: 0,58-0,88). Conclusão: $O$ homicídio está inserido em um quadro de complexas questões sociais, dentre as quais se insere o envolvimento com drogas ilícitas, e é mais prevalente entre indivíduos com histórico de encarceramento e menos prevalente entre os de mais de 30 anos.
\end{abstract}

Palavras-chave: violência; mortalidade; homicídio.

\begin{abstract}
Introduction: Homicide is a public health problem in Brazil that is often related to illicit drugs. Objective: To characterize homicides and aspects associated with the use of illicit drugs. Methods: A cross-sectional study with a quantitative approach carried out in Joao Pessoa, in the state of Paraiba, Brazil, in 2014. 424 homicide victims whose data were collected through a specific instrument were considered, analyzed descriptively and analytically with the aid of statistical software, with Chi Squared Test and Robust Poisson Regression, considering a confidence level of $95 \%$ and statistical significance when $p<0.05$. Results: Prevalence of homicide victims (93.2\%), non-whites (97.2\%), with up to 30 years, considering the age groups below 20 years (22.6\%), 20-24 $(26.7 \%)$ and 25 to 29 (18.8\%), with less than seven years of study (67.5\%), with a history of involvement with illicit drugs (72.5\%) and incarceration (59.7\%). There was an association between involvement with illicit drugs with sex $(p=0.037)$, age $(p=0.002)$ and history of incarceration $(p<0.001)$. There was a $67 \%$ higher prevalence of involvement with illicit drugs among those with a history of incarceration (PR: 1.67, 95\% Cl: 1.44-1.94) and $28 \%$ lower among individuals aged 30 years or older (PR: $0.72, \mathrm{Cl}$ : 0.58-0.88). Conclusion: Homicide is part of a complex set of social issues, including involvement with illicit drugs, which is more prevalent among young adults and individuals with a history of incarceration.
\end{abstract}

Keywords: violence; mortality; homicide.

Recebido em: 04/09/2018

Revisado em: 18/01/2019

Aprovado em: 04/02/2019

Autor para correspondência: Alline Oliveira do Nascimento Veloso - Universidade Estadual da Paraíba - Rua das Baraúnas, 351 - Bairro Universitário CEP: 58429-500 - Campina Grande (PB), Brasil. E-mail: lilionvjp@gmail.com

Conflito de interesses: nada a declarar. 


\section{INTRODUÇÃO}

O homicídio, agravo interpessoal infringido por qualquer meio e com desfecho letal, possui elevado impacto social, e não por acaso tem sido considerado o principal indicador de violência; que afeta de forma importante populações de um sem-número de países, sobretudo os periféricos e em desenvolvimento ${ }^{1}$.

Na América Latina, no período de 1999 a 2009, depreendeu-se que os países com as maiores taxas de homicídio, considerando uma base de 100 mil habitantes, foram El Salvador $(62,9)$, Guatemala $(51,2)$, Colômbia $(42,5)$, Venezuela $(33,2)$ e Porto Rico $(25,8)$. No mesmo período, verificou-se que no caso do Brasil, embora com uma taxa inferior àqueles $(25,2)$, a qual se manteve relativamente estável, ainda é cerca de quatro vezes superior à média mundial ${ }^{2,3}$.

Com efeito, considerando o contexto brasileiro, em 2014, ano do presente estudo, foram registrados 59.681 homicídios, e, no estado da Paraíba, na região nordeste, a qual tem apresentado, sistematicamente, elevação nos citados agravos, foram reportados 1.522, o que representou 39,3 indivíduos mortos para cada 100 mil habitantes. Com destaque para a sua capital, João Pessoa, cenário do presente estudo, comportando pouco mais de um terço dos óbitos, resultado que a fez ocupar importante colocação no ranking entre todas as outras capitais ${ }^{4,5}$.

Destarte os aspectos ora apresentados, é certo que o homicídio é a expressão da violência que tem demandado atenção, sobretudo porque não atinge a população de forma homogênea, mas antes, sobremaneira desigual, porquanto se tem percebido, na totalidade dos estudos com essas temáticas, e ainda que se tenham reportado de forma consistente o crescimento das estatísticas de feminicídio ${ }^{6}$, sua maior incidência entre homens, jovens, negros, de baixa escolaridade ${ }^{7,8}$, provenientes das periferias dos grandes centros urbanos ${ }^{9}$, e entre aqueles com algum tipo de envolvimento com drogas ilícitas ${ }^{10}$. Revela-se, pois, uma série de fatores relacionados ao agravo em tela, em particular aqueles de ordem socioeconômica e com a questão do uso e tráfico de entorpecentes, principalmente ${ }^{11,12}$.

Assim sendo, se por um lado o incremento em estudos com a temática de homicídios pode contribuir para reforçar a intrínseca relação existente entre estes e aspectos socioeconômicos amplamente conhecidos, por outro, quando inclui a temática de drogas ilícitas, tal como se fez no presente estudo, mostra-se ainda mais relevante, na medida em que pode contribuir com discussões e operacionalização de ações a respeito de questões como legalização e descriminalização, as quais certamente estão na ordem do dia.

Este estudo, em particular, que traz como mérito o uso de inquéritos policiais, e não dados de sistemas de informação, porquanto permite explorar aspectos não passíveis de serem aferidas através daqueles, além do contributo teórico, possui ainda aplicabilidade prática. Isso porque com a maior compreensão destes agravos pode-se contribuir para além do contexto da segurança pública, e ser importante para subsidiar o delineamento de políticas intersetoriais que envolvam diferentes atores políticos, sobretudo no contexto de crise econômica e de austeridade fiscal em voga, que certamente impactará mais ainda a realidade em tela nas próximas décadas.

Diante do exposto, este estudo tem por objetivo caracterizar os homicídios e os aspectos associados ao uso de drogas ilícitas em João Pessoa, uma das capitais do Nordeste do Brasil.

\section{MÉTODOS}

Estudo transversal, com abordagem quantitativa, realizado na cidade de João Pessoa, capital do estado da Paraíba, situada na região Nordeste do Brasil, em 2014.

A base para o estudo foram os inquéritos policiais de 424 vítimas de homicídio, agravo interpessoal infringido por qualquer meio e com desfecho letal, realizados pela Polícia Civil naquela cidade, no ano em questão. Tais documentos estão concentrados na Delegacia de Crimes contra a Pessoa, os quais constituem instrumentos formais de investigação e representam o conjunto de diligências produzidas por Investigadores de Polícia e Peritos Criminais, organizados pelo Escrivão de Polícia, e presidido pelo Delegado de Polícia, com o objetivo de apurar o fato criminoso e descobrir sua autoria ${ }^{13}$.

Considerando o exposto e após a apresentação do projeto de pesquisa e da devida autorização institucional para o seu prosseguimento, por meio da autoridade competente, os pesquisadores envolvidos inseriram-se no cenário de estudo e encaminharam a coleta de dados. Utilizou-se um instrumento próprio, do tipo formulário, tendo como base principalmente os dados constantes nos documentos que compõe os inquéritos policiais de cada uma de tantas quantas foram às vítimas de homicídio, e que, portanto, poderiam ser captados, a saber: o Boletim de Ocorrência, os Termo de Declaração de Familiares, o Laudo Cadavérico e o Laudo de Local de Crime.

A partir dos documentos retro mencionados, foram elaboradas questões que contemplaram variáveis sociodemográficas gerais: sexo, idade, raça/cor, grau de escolaridade e estado civil; variáveis relacionadas aos homicídios: dia da semana, turno, local do crime, local do óbito, meio utilizado, quantidade de lesões e região do corpo acometida; além de variáveis sobre a utilização de drogas ilícitas e histórico de encarceramento.

Os dados foram tabulados e processados em banco de dados único, elaborado com auxílio do Software Statistical Package for the Social Sciences (SPSS), versão 20.0, a partir do qual se procedeu a análise estatística, do ponto de vista descritivo, sendo aferidos frequência absoluta e relativa, e analítico, a partir de modelos univariado e múltiplo, considerando significância estatística quando $\mathrm{p}<0,05$ e nível de confiança de $95 \%$.

$\mathrm{Na}$ análise univariada, foram investigados, através do teste de Qui-Quadrado, os fatores associados ao envolvimento com de 
drogas ilícitas, variável dependente, considerando como variáveis independentes o sexo: feminino e masculino; a idade: $<20$ anos, 20 a 24 anos, 25 a 29 anos e $\geq 30$ anos; a escolaridade: $<7$ anos de estudo e $\geq 7$ anos de estudo; a cor: brancos e não brancos; e o histórico de encarceramento: sim e não; calculando-se o p-valor.

Para a análise múltipla, realizada através da Regressão Robusta de Poisson, desenvolveu-se um modelo para investigação dos fatores associados ao envolvimento com de drogas ilícitas, variável dependente, com ajuste para variáveis independentes, tais como o sexo: feminino e masculino; a idade: $<20$ anos, 20 a 24 anos, 25 a 29 anos e $\geq 30$ anos, a escolaridade: $<7$ anos de estudo e $\geq 7$ anos de estudo; a cor: brancos e não brancos; e o histórico de encarceramento: sim e não; calculando-se a Razão de Prevalência ajustada (RPa) e Intervalos de Confiança (IC).

Por questões ético-legais, a pesquisa foi realizada em observância aos princípios e diretrizes apontados na Resolução No 466 de 2012 do Conselho Nacional de Saúde, sendo registrada na Plataforma Brasil e aprovada pelo Comitê de Ética em Pesquisa da Universidade Estadual da Paraíba, sob o Certificado de Apresentação para Apreciação Ética (CAAE) de No 53324316.7.00005187.

\section{RESULTADOS}

Com um total de 424 vítimas de homicídios, contabilizados em João Pessoa, no estado da Paraíba, cenário deste estudo, em 2014, uma média mensal de $35,3,1,1$ por dia e uma taxa de 52,8 para 100 mil habitantes, verificou-se a predominância de homens $(93,2 \%)$, não brancos $(97,2 \%)$, com até 30 anos, considerando as faixas etárias de menor de 20 anos (22,6\%), de 19 a 24 (26,7\%) e 25 a $29(16,8 \%)$, com menos de sete anos de estudo (67,5\%), histórico de envolvimento com drogas (72,5\%), e mais de um terço com histórico de reclusão (40,3\%) (Tabela 1$)$.

No que se refere às características dos agravos, os homicídios, verificou-se que a maioria ocorreu durante dias úteis (67\%), em período noturno $(56,4 \%)$ e em via pública $(75 \%)$, por meio de arma de fogo $(86,8 \%)$, com ocorrência de múltiplas lesões $(84,2 \%)$, e com óbito declarado ainda no local do crime (82,5\%) (Tabela 2).

Prosseguindo-se com as características dos homicídios, destacaram-se os ferimentos nas regiões da cabeça $(60,8 \%)$ e do tórax $(55,4 \%)$ (Tabela 3$)$.

Como parte expressiva das vítimas possuíam histórico de envolvimento com drogas ilícitas, realizou-se análise univariada considerando-o como variável dependente, e aspectos sociodemográficos como independentes, revelando-se significância estatística com o sexo $(\mathrm{p}=0,037)$, a idade $(\mathrm{p}=0,002)$, e o histórico de encarceramento $(\mathrm{p}<0,001)$ (Tabela 4$)$.

Verificou-se que a prevalência de envolvimento com drogas ilícitas foi maior $16 \%$ em indivíduos do sexo masculino (RP: 1,16; IC95\%: 0,79-1,73), 19\% em não brancos (RP: 1,19; IC95\%: 0.76-1,86) e $67 \%$ naqueles que com histórico de encarceramento (RP: 1,67; IC95\%: 1,44-1,94), sendo este com significância estatística ( $\mathrm{p}<0,001)$.
Tabela 1: Distribuição das vítimas de homicídio doloso, segundo variáveis sociodemográficas. João Pessoa (PB), 2015.

\begin{tabular}{|c|c|c|c|}
\hline \multicolumn{2}{|l|}{ Variáveis } & $\mathbf{N}$ & $\%$ \\
\hline \multirow{2}{*}{ Sexo } & Feminino & 29 & 6,8 \\
\hline & Masculino & 395 & 93,2 \\
\hline \multirow{4}{*}{ Faixa etária } & $<20$ & 96 & 22,6 \\
\hline & $20-24$ & 113 & 26,7 \\
\hline & $25-29$ & 71 & 16,8 \\
\hline & $\geq 30$ & 144 & 33,9 \\
\hline \multirow{2}{*}{ Estado Civil } & Sem companheiro & 299 & 70,5 \\
\hline & Com companheiro & 125 & 29,5 \\
\hline \multirow{2}{*}{ Raça/Cor } & Não Branco & 412 & 97,2 \\
\hline & Branco & 12 & 2,8 \\
\hline \multirow{2}{*}{ Escolaridade } & $<7$ anos de estudo & 286 & 67,5 \\
\hline & $\geq 7$ anos de estudo & 138 & 32,5 \\
\hline \multirow{2}{*}{ Envolvimento com drogas ilícitas* } & Sim & 242 & 72,5 \\
\hline & Não & 92 & 27,5 \\
\hline \multirow{2}{*}{ Histórico de encarceramento** } & Não & 182 & 59,7 \\
\hline & Sim & 123 & 40,3 \\
\hline
\end{tabular}

Tabela 2: Distribuição das vítimas de homicídio doloso segundo as características do agravo. João Pessoa (PB), 2015.

\begin{tabular}{|l|c|c|c|}
\hline Variáveis & Uma & N & $\%$ \\
\hline Número de vítimas & Duas & 38 & 91,0 \\
\hline \multirow{2}{*}{ Dia da Semana } & Dias úteis & 284 & 67,0 \\
\hline \multirow{3}{*}{ Turno } & Final de semana & 140 & 33,0 \\
\hline \multirow{3}{*}{ Local do Crime } & Diurno & 185 & 43,6 \\
\hline \multirow{3}{*}{ Meio utilizado } & Noturno & 239 & 56,4 \\
\hline \multirow{2}{*}{ Quantidade de Lesóblica } & 318 & 75,0 \\
\hline \multirow{2}{*}{ Local do óbito } & Residência & 64 & 15,1 \\
\hline & Outros & 42 & 9,9 \\
\hline & Arma de fogo & 361 & 86,8 \\
\hline & Arma branca & 42 & 9,9 \\
\hline & Outros* & 14 & 3,3 \\
\hline & Múltiplas & 357 & 84,2 \\
\hline & Única & 67 & 15,8 \\
\hline & No local do crime & 350 & 82,5 \\
\hline & No transporte & 12 & 2,9 \\
\hline & No hospital & 62 & 14,6 \\
\hline
\end{tabular}

*intoxicação, asfixia, explosão, etc.

Tabela 3: Distribuição das vítimas de homicídio doloso por região do corpo acometida segundo e sexo. João Pessoa (PB), 2015.

\begin{tabular}{|c|c|c|c|c|c|c|}
\hline \multirow{3}{*}{ Região do corpo } & \multicolumn{4}{|c|}{ Sexo } & \multirow{2}{*}{\multicolumn{2}{|c|}{ Total }} \\
\hline & \multicolumn{2}{|c|}{ Feminino } & \multicolumn{2}{|c|}{ Masculino } & & \\
\hline & $\mathbf{N}$ & $\%$ & N & $\%$ & $\mathbf{N}$ & $\%$ \\
\hline Cabeça & 14 & 48,3 & 244 & 61,8 & 258 & 60,8 \\
\hline Face & 6 & 20,7 & 75 & 19,0 & 81 & 19,1 \\
\hline Pescoço & 6 & 20,7 & 58 & 14,7 & 64 & 15,1 \\
\hline Tórax & 13 & 44,8 & 222 & 56,2 & 235 & 55,4 \\
\hline Abdômen & 4 & 13,8 & 95 & 24,1 & 99 & 23,3 \\
\hline Dorso & 5 & 17,2 & 85 & 21,5 & 90 & 21,2 \\
\hline Genitálias & 1 & 3,4 & 7 & 1,8 & 8 & 1,9 \\
\hline Glúteos & 0 & 0,0 & 15 & 3,8 & 15 & 3,5 \\
\hline MMSS* & 8 & 27,6 & 133 & 33,7 & 141 & 33,3 \\
\hline $\mathrm{MMII}^{\star \star}$ & 3 & 10,3 & 63 & 15,9 & 66 & 15,6 \\
\hline
\end{tabular}

*Membros Superiores; ${ }^{\star \star}$ Membros Inferiores 
Ademais, constatou-se que a prevalência de envolvimento com drogas ilícitas foi $2 \%$ menor entre aqueles que possuíam mais de sete anos de estudo (RP: 0,98; IC95\%: 0,84-1,15) e menos prevalente tanto quanto maior a faixa de idade, sendo $28 \%$ menos prevalente entre aqueles com 30 ou mais anos (RP: 0,72; IC95\%: 0,58-0,88), achado estatisticamente significante $(\mathrm{p}=0,002)$ (Tabela 5$)$.

\section{DISCUSSÃO}

O contexto epidemiológico do Brasil é marcado por uma tripla carga de doenças e agravos com importância sanitária ${ }^{14}$, a qual inclui doenças infectocontagiosas e parasitárias, e principalmente, afecções não transmissíveis e os agravos externos e a violência; isto pela magnitude que vem assumindo, sobretudo com o homicídio, cerca de quatro vezes superior à média mundial² .

Conforme se depreendeu, houve uma média mensal de 35,3 homicídios, 52,8 indivíduos mortos para cada 100 mil habitantes, números expressivos quando considerados os últimos levantamentos oficiais reportados no âmbito brasileiro, que dão conta de uma taxa de 25,8, e em particular no estado em que João Pessoa, cenário deste estudo, está situado, na Paraíba, com 39,3. De pronto, pode-se constatar o destaque daquela cidade no âmbito estadual, mas também nacionalmente, o que não por acaso a fez ocupar a quarta colocação, entre todas as outras capitais ${ }^{4,5}$.

Embora haja muito se conheça que os homicídios, como os mencionados, resultam, em linhas gerais, da combinação de uma multiplicidade de fatores, quer individuais, referentes às vítimas e agressores, quer externas a elas ${ }^{7,15,16}$, torna-se forçoso, não obstante, reiterar que não se distribuem homogeneamente em toda a população, e embora se constate que o feminicídio, ou a morte de mulheres por questões de gênero ou por violência doméstica, tem cada vez mais se evidenciado ${ }^{6}$, eles estão concentrados em homens, jovens, não-brancos e de baixa escolaridade, conforme há tempos tem se demonstrado em outros resultados ${ }^{17,18,19,20,21,22,23}$, com os quais aqueles aqui apresentados possuem consistência.

Destarte, urge evidenciar, pois, a notória concentração das vítimas de homicídios em uma população de particular características demográficas e socioeconômicas, oriundas, sobretudo da região Nordeste

Tabela 4: Distribuição de vítimas de homicídio doloso segundo variáveis sociodemográficas e envolvimento com drogas ilícitas. João Pessoa (PB), 2015

\begin{tabular}{|c|c|c|c|c|c|c|}
\hline \multirow{2}{*}{\multicolumn{2}{|c|}{ Variáveis Independentes }} & \multicolumn{4}{|c|}{ Envolvimento da vítima com drogas ilícitas* } & \multirow{4}{*}{$\begin{array}{c}\text { P valor } \\
0,037\end{array}$} \\
\hline & & \multicolumn{2}{|c|}{ Sim } & \multicolumn{2}{|c|}{ Não } & \\
\hline & & $\mathbf{n}$ & $\%$ & $\mathbf{n}$ & $\%$ & \\
\hline \multirow{2}{*}{ Sexo } & Masculino & 229 & 73,9 & 81 & 26,1 & \\
\hline & Feminino & 13 & 54,2 & 11 & 45,8 & \\
\hline \multirow{4}{*}{ Idade } & $<20$ & 57 & 77,1 & 17 & 22,9 & 0,002 \\
\hline & $20-24$ & 73 & 81,1 & 17 & 18,9 & \\
\hline & $20-29$ & 44 & 78,6 & 12 & 21,4 & \\
\hline & $\geq 30$ & 68 & 59,7 & 46 & 40,1 & \\
\hline \multirow[t]{2}{*}{ Raça/Cor } & Branco & 8 & 66,7 & 4 & 33,3 & 0,648 \\
\hline & Não-Branco & 234 & 72,7 & 88 & 27,5 & \\
\hline \multirow{2}{*}{ Escolaridade } & $<7$ anos de estudo & 166 & 74,1 & 58 & 69,1 & 0,335 \\
\hline & $\geq 7$ anos de estudo & 76 & 25,9 & 34 & 30,9 & \\
\hline \multirow{2}{*}{$\begin{array}{l}\text { Histórico de } \\
\text { encarceramento }\end{array}$} & Sim & 108 & 90,0 & 12 & 10,0 & $<0,001^{\star \star}$ \\
\hline & Não & 92 & 54,1 & 78 & 45,9 & \\
\hline
\end{tabular}

${ }^{*} \mathrm{n}=334 ;{ }^{* *}$ Qui-quadrado

Tabela 5: Distribuição de vítimas de homicídio doloso segundo variáveis sociodemográficas e envolvimento com drogas ilícitas. João Pessoa (PB), 2015.

\begin{tabular}{|c|c|c|c|c|}
\hline \multirow{2}{*}{\multicolumn{2}{|c|}{ Variáveis Independentes }} & \multicolumn{3}{|c|}{ Utilização de drogas ilícitas } \\
\hline & & RP ajustado* & IC95\% & $\mathbf{P}$ \\
\hline \multirow{2}{*}{ Sexo } & Mulher & 1 & & \\
\hline & Homem & 1,16 & $0,79-1,73$ & 0,435 \\
\hline \multirow{4}{*}{ Idade } & $<20$ & 1 & & \\
\hline & $20-24$ & 0,99 & $0,82-1,18$ & 0,880 \\
\hline & $20-29$ & 0,90 & $0,74-1,11$ & 0,339 \\
\hline & $\geq 30$ & 0,72 & $0,58-0,88$ & 0,002 \\
\hline \multirow{2}{*}{ Raça/Cor } & Branco & 1 & & \\
\hline & Não-Branco & 1,19 & $0.76-1.86$ & 0,443 \\
\hline \multirow{2}{*}{ Escolaridade } & $<7$ anos de estudo & 1 & & \\
\hline & $\geq 7$ anos de estudo & 0,98 & $0,84-1,15$ & 0,827 \\
\hline \multirow{2}{*}{$\begin{array}{l}\text { Histórico de } \\
\text { Encarceramento }\end{array}$} & Não & 1 & & \\
\hline & Sim & 1,67 & $1,44-1,94$ & $<0,001$ \\
\hline
\end{tabular}

*Regressão Robusta de Poisson 
brasileira, na qual aqueles têm sido reportados em proporções cada vez mais elevadas. Acredita-se que o crescimento populacional dos seus centros urbanos, principalmente das capitais, de um lado, não tem se seguido de uma adequada estruturação para parte de seus habitantes, os quais estão em não raras realidades marginalizados ${ }^{4,24}, \mathrm{e}$ do outro, tem atraído algumas facções criminosas de diferentes locais do país e o desenvolvimento local de outras, ambas as realidades que podem cooperar para o aumento da violência.

Embora se reconheça a ampla realidade em que o homicídio se insere, no contexto mais geral das características de sua ocorrência, entre as quais um sem número de questões que, individualmente, poderiam ser amplamente estudadas e discutidas, como aquelas aqui já colocadas - sexo, idade, escolaridade e cor - que carecem ser analisadas não por si mesmas, mas porque apontam para questões sociais amplas, como a desigualdade, sobretudo as duas últimas. Ainda que amplamente conhecida e debatida, tal impressão continua a demandar importantes reflexões, sobretudo pelo grau de naturalização a que chegou ${ }^{4}$.

Sobre o exposto, a partir de análise dos homicídios em quase uma década, entre os anos 2000 e 2009, verifica-se que aspectos relacionados à escolaridade, assim como a cor, podem ser fatores concorrentes para maior vitimização por estes agravos ${ }^{18}$, o que também foi discutido a partir de outras análises ${ }^{25}$. No entanto, segundo o que concluem os seus autores, ainda que se considerem os mais e os menos escolarizados, aqueles agravos foram mais incidentes entre indivíduos não-brancos quando comparados com os brancos, e sugere, portanto, que o segundo aspecto explica parte importante da sua ocorrência ${ }^{25}$.

Ainda considerando o contexto mais geral de características da ocorrência dos homicídios, além daquelas anteriormente colocadas, é importante constatar que as vítimas majoritariamente morreram no local do crime, o que pode ser explicado, em parte, se considerado que a maioria sofreu múltiplas lesões, sobretudo em regiões em que estão localizados os órgãos vitais, como tórax e cabeça, conforme já se constatou ${ }^{26}$; sendo a arma de fogo o principal instrumento envolvido, aspecto observado em análises precedentes ${ }^{26,27,28}$.

De pronto, no contexto em tela, pode-se supor que não obstante os homicídios sejam, de fato, um importante problema de saúde pública, na realidade sanitária global, e em particular na brasileira, atualmente valeria dizer ainda que se tratam de agravos que além de afetarem indivíduos com peculiares características, conforme exposto anteriormente, ocorrem de uma singular forma, porquanto envolve alto grau de violência, quer pelo instrumento utilizado pelo agressor, a arma de fogo, quer pelas características da morte dos indivíduos, em geral no cenário em que foram vitimados, com poucas chances de serem socorridos.

Em um contexto de centralidade da arma de fogo em homicídios, coloca-se em questão o próprio Estatuto do Desarmamento, que apesar de datar de mais de uma década, pode não ter obtido o impacto social esperado em todos os grupos populacionais.
Assim, ainda que se perceba um ponto de inflexão na incidência de mortes, sem recorte por categoria, bem como considerando apenas os que ocorreram por aquelas que ocorreram por disparo de arma de fogo, em seu primeiro ano de implementação, em 2005, a presumida estabilização nos anos posteriores só ocorreu no primeiro grupo e não no segundo ${ }^{5}$.

Destarte os homicídios por disparos de arma de fogo apresentaram consistente elevação ${ }^{5}$, ainda que em um contexto de legislação mais restritiva, com o Estatuto do Desarmamento, de ampliação de políticas sociais, de distribuição de renda, habitação, acesso à educação e saúde, sobretudo na primeira década dos anos 2000, e, ainda, de aumento da proporção de idosos, menos expostos a agravos externos, como acidentes e violências, e redução de jovens e adultos de 15 a 29 anos, que ao contrário são mais vulneráveis $^{29}$. Segundo dados demográficos, entre 2005, ano do estatuto, e 2015, a proporção dos primeiros aumentou cerca de $25 \%$, e a do segundo, ao contrário, diminuiu $10 \%$ no período ${ }^{29}$.

Assim, o acesso às armas de fogo continua amplamente difundido, sobretudo entre as facções criminosas, e, pode-se supor, tem vitimado adolescentes e adultos jovens com a violência, sobretudo em bairros pobres das grandes cidades ${ }^{16,30}$, como as capitais. $\hat{E}$ possível atribuir isso, de um lado, a presumida baixa efetividade do Estatuto do Desarmamento, sobretudo pela carência de ações intersetoriais de suporte; bem como ao fato de que a ampliação de políticas sociais, difundidas na primeira década dos anos 2000 , apesar de importantes, crê-se que não foram suficientes para impactar de forma mais concreta os indicadores de homicídios.

Além da arma de fogo, o envolvimento com drogas ilícitas como maconha, crack e cocaína, é uma questão patente entre as vítimas de homicídio, porquanto se depreende que um quantitativo relevante utilizava uma ou algumas destas substâncias ${ }^{10,31}$. Tais observações não estão circunscritas apenas no cenário investigado, do Nordeste, mas também externos a ele, como no sudeste ${ }^{21,29}$, e mais ainda, também no plano internacional, porquanto os aspectos que circunscrevem o consumo das citadas substâncias, sobretudo o tráfico, está correlacionado com maior risco para o tipo de agravo em tela ${ }^{27,32}$.

Destarte, o contexto que circunscreve o consumo e tráfico de drogas ilícitas é reconhecido atualmente como um dos principais fatores de risco para a morte por homicídios ${ }^{33,34}$, e, considerando as características associadas, conforme se depreendeu também neste estudo - idade, sexo, e histórico de reclusão- revelam-se aspectos que precisam ser considerados, sobretudo no cenário atual, em que há um movimento internacional de indivíduos que, através de organizações diversas, tencionam fortemente o debate sobre legalização e descriminalização do uso destas substancias, para que se possa qualificar os posicionamentos.

Conforme se observou, quando considerado o ajuste de variáveis em um modelo de regressão, idade e histórico de reclusão continuaram estatisticamente associadas ao envolvimento com drogas ilícitas, observando-se que a prevalência desta condição foi maior entre os que já foram encarcerados e menor tanto quanto maior 
a faixa-etária, principalmente para maiores de 30 anos. Supõe-se que a associação entre o uso destas substâncias e comportamentos violentos dos usuários possa ser explicado, de um lado, pela necessidade de incidir em crimes através dos quais obtenham recursos necessários à aquisição destas, e do outro, e talvez principalmente, por sua relação mais próxima com o narcotráfico, cujos agentes tem recrutado indivíduos cada vez mais jovens ${ }^{30,32,35,36}$, ambas realidades que não raro resultam em morte ou encarceramento.

Em um cenário de um exacerbado proibicionismo e, por conseguinte, ausência de regulação, para drogas além de tabaco e álcool, e profunda desigualdade social, faz destas substâncias importantes forças motrizes da criminalidade que tem no tráfico um dos seus seguimentos mais rentáveis, e que acaba por recrutar e vitimar, sobretudo pelo homicídio, de um modo geral, exatamente aqueles que são as maiores vítimas de iniquidades ora colocadas: negros, adultos-jovens, moradores de periferias, indivíduos com baixa instrução e de reduzido nível econômico.

Nesse contexto, não por acaso constata-se que em cenários de superação das iniquidades, com a melhoria das condições sociais da população, aumento da renda per capita, a diminuição das taxas de desemprego, a diminuição das desigualdades de renda, o aumento das taxas de cobertura e frequência escolar, bem como a eficácia das políticas de prevenção e segurança pública, as taxas de homicídios são menores ${ }^{37}$.

Não obstante, na realidade brasileira, uma das 10 mais desiguais do mundo, e que ocupa $\mathrm{o} 79^{\circ}$ posição no ranking de IDH, em não raros contextos, vê-se o exato oposto, com ampliação da pobreza e da pobreza extrema, na qual estão inseridas 54 milhões e $15 \mathrm{mi}$ lhões de indivíduos, algo em torno de $26,5 \%$ e $7,4 \%$ da população, respectivamente; quadro ainda pior quando considerado apenas o Nordeste ${ }^{38,39}$. Entre os mais jovens, crianças e adolescentes, as desigualdades impactam a vida de seis em cada 10 indivíduos, porquanto $61 \%$ convive com privações de direitos fundamentais, como educação, saneamento, informação, moradia, água potável e proteção contra o trabalho infantil ${ }^{40}$.

Ademais, valeria destacar, além dos pontos retro mencionados, também a ampliação de ações de prevenção ao homicídio, a partir de uma cultura da paz que atinjam a todos os grupos sociais, com uma maior discussão sobre posse e porte de armas de fogo e a utilização de drogas ilícitas, aliadas a políticas públicas efetivas contra a impunidade, sobretudo no tráfico, conforme apontado em importante estudo sobre a questão ${ }^{30}$. Estas iniciativas podem ser potencialmente benéficas, para reduzir os números de homicídios e os efeitos deletérios a sociedade, mas que só surtirão efeito se consideradas como parte integrantes de uma complexa estratégia intersetorial.

No que se refere às limitações deste estudo, considera-se que pesquisas sobre homicídios são sempre complexas, sobretudo em estudos transversais, uma vez que múltiplos fatores, incluindo desfecho e exposições, são avaliados concomitantemente, o que não permite estabelecer relações causais. Ademais, o envolvimento com drogas ilícitas, também abordado, é um desfecho multideterminado, de modo que é pouco provável o controle de todos os fatores que o influenciam numa análise. Esse estudo, pois, não trata de fatores que causam o homicídio ou a utilização de drogas ilícitas, mas pretende diagnosticar, no cenário estudado, o contexto mais geral em que ocorrem. Por fim, a diferença entre alguns dos dados aqui apresentados e aqueles disponibilizados pelo Sistema de Informação de Mortalidade podem divergir minimamente, porquanto após o envio destes mesmos dados ao Ministério da Saúde, não é raro que isso implique em algumas alterações em virtude de correções realizadas, inclusive de redistribuição dos óbitos pelo local de residência.

\section{REFERÊNCIAS}

1. Costa FAMM, Trindade RFC, Santos CB. Mortes por homicídios: série histórica. Rev Latino-Am Enfermagem. 2014;22(6):1017-25. http://dx.doi.org/10.1590/0104-1169.3603.2511

2. United Nations Office on Drugsand Crime (UNODC). 2011 Global study on homicide. trends, contexts, data. Viena: UNODC, 2014.

3. Gawryszewski VP, Sanhueza A, Martinez-Piedra R, Escamilla JA, Souza MFM. Homicídios na região da Américas: magnitude, distribuição e tendências, 1999-2009. Ciênc Saúde Coletiva. 2012;17(12):3171-82.

http://dx.doi.org/10.1590/S1413-81232012001200003

4. Cerqueira D, Lima RS, Bueno S, Valencia LV, Hanashiro O, Machado PHG, et al. Atlas da violência 2017. Rio de Janeiro: IPEA, 2017.

5. Brasil. Ministério da Saúde. Data SUS. Sistema de mortalidade (SIM). Disponível em: http://tabnet.datasus.gov.br/cgi/deftohtm. exe?sim/cnv/ext10pb.def. Acesso em: 8 out. 2013.

6. United Nations Office on Drugs and Crime (UNODC). Global Study on Homicide. Disponível em: https://www.unodc.org/documents/ data-and-analysis/GSH2018/GSH18 Gender-related killing of women and girls.pdf. Acesso em: 18 jan. 2018.
7. Minayo MCS, Constantino P. An ecosysthemic view of homicide. Ciênc Saúde Coletiva. 2012;17(12):3269-78.

http://dx.doi.org/10.1590/S1413-81232012001200012

8. Drumond EF, Hang-Costa TA, Souza HNF. Presença de álcool em adolescentes vítimas de homicídios em Belo Horizonte 20052009. Rev Min Enferm. 2014;18(2):272-7.

http://www.dx.doi.org/10.5935/1415-2762.20140021

9. Jimenez L, Frasseto FA. Face da morte: a lei em conflito com o adolescente. Psicol Soc. 2015;27(2):404-14.

http://dx.doi.org/10.1590/1807-03102015v27n2p404

10. Swedler DI, Simmons MM, Dominici F, Hemenway D. Firearm Prevalence and Homicides of Law Enforcement Officers in the United States. Am J Public Health. 2015;105(10):2042-8. http://dx.doi.org/10.2105/AJPH.2015.302749

11. Rubanzana W, Ntaganira J, Freeman MD, Gauthier BLH. Risk factors for homicide victimization in post-genocide Rwanda: a population based case- control study. BMC Public Health. 2015;15:809. http://dx.doi.org/10.1186/s12889-015-2145-z

12. Poveda AC. Violence and economic development in Colombian cities: a dynamic panel data analysis. J Int Dev. 2012;24(7):809-27. https://dx.doi.org/10.1002/jid.2819 
13. Tourinho Filho, Costa F. Manual de processo penal. 16ed. São Paulo: Saraiva, 2013.

14. Mendes EV. As redes de atenção à saúde. Ciênc Saude Coletiva. 2010;15(5):2297-305. http://dx.doi.org/10.1590/S1413-81232010000500005

15. Kleinschmitt SC, Wadi YM, Staduto JA. Analise espacial dos homicídios no estado do Paraná. Rev Desenvolv Reg. 2012;17(3):257-90.

http://dx.doi.org/10.17058/redes.v17i3.1203

16. Zilli LF. O "mundo do crime" e a "lei da favela": aspectos simbólicos da violência de gangues na região metropolitana de Belo Horizonte. Etnográfica. 2015;19(3):463-87.

17. Souza TO, Souza ER, Pinto LW. Evolução da mortalidade por homicídios no Estado da Bahia, Brasil, no período de 1996 a 2010. Ciênc Saúde Coletiva. 2014;19(4):1889-1900. http://dx.doi.org/10.1590/1413-81232014196.04772013

18. Soares Filho AM. Homicide victimization according to racial characteristics in Brazil. Rev Saúde Pública. 2011;45(4):745-455. http://dx.doi.org/10.1590/s0034-89102011005000045

19. Acevedo CYR, Dueñas LPZ, Castañeda-Porras O. Lesiones fatales en adolescentes, Casanare-Colombia 2011-2013. Rev Med Risaralda. 2016;22(1):18-29.

20. Minayo MCS. Seis características das mortes violentas no Brasil. Rev Bras Est Pop. 2009;26(1):135-40. http://dx.doi.org/10.1590/S0102-30982009000100010

21. Alves WA, Correia DS, Barbosa LLB, Lopes LM, Melânia MIASM. Violência letal em Maceió-AL: estudo descritivo sobre homicídios, 2007-2012. Epidemiol Serv Saúde. 2014;23(4):731-40. http://dx.doi.org/10.5123/S1679-49742014000400015

22. Bastos MJRP, Pereira JA, Smarzarro DC, Costa EF, Bossanel RCL, Oliosa DMS, et al. Ecological analysis of acidentes and lethal violence in Vitória, Southeastern Brazil. Rev Saúde Pública. 2009;43(1):123-32. http://dx.doi.org/10.1590/S0034-89102009000100016

23. Lima ALB, Lima KC, Maia LTS, Oliveira TC. Tendência crescente de violência homicida na região metropolitana de Natal-RN, Brasil. Rev Ciênc Plural. 2015;1(2):19-28.

24. Costa AC, Marguti BO. Atlas de Vulnerabilidade Social nos Municípios Brasileiros. Rio de Janeiro: IPEA, 2015.

25. Soares Filho AM, Souza MFM, Gazal-Carvalho C, Malta DC, Alencar AP, Silva MMA, et al. Análise da mortalidade por homicídios no Brasil. Epidemiol Serv Saude. 2007;16(1):7-18. http://dx.doi.org/10.5123/S1679-49742007000100002

26. Campos MEAL, Ferreira LOC, Barros MDA, Silva HL. Mortes por homicídio em município da Região Nordeste do Brasil, 2004-2006 a partir de dados policiais. Epidemiol Serv Saúde. 2011;20(2):151-9. http://dx.doi.org/10.5123/S1679-49742011000200004

27. Trindade RFC, Costa FAMM, Silvia PPAC, Caminiti GB, Santos CB. Mapa dos homicídios por arma de fogo: perfil das vítimas e das agressões. Rev Esc Enferm USP. 2015;49(5):748-55. https://doi.org/10.1590/S0080-623420150000500006
28. Zavala-Zegarra DE, López-Charneco M, Garcia-Rivera EJ, Concha-Eastman A, Rodriguez JF, Conte-Miller M. Geographic distribution of risk of death due to homicide in Puerto Rico, 2001-2010. Rev Panam Salud Pública 2012;32(5):321-9

https://doi.org/10.1590/s1020-49892012001100001

29. Brasil. Ministério da Saúde. DATASUS: estimativas população: município, sexo e idade 2000 - 2015. Disponível em: http:// www2.datasus.gov.br/DATASUS/index.php?area=0206\&id=6942. Acesso em: 18 jan 2018

30. Barcellos C, Zalluar A. Homicídios e disputas territoriais nas favelas do Rio de Janeiro. Rev Saúde Pública. 2014;48(1):94-102 http://dx.doi.org/10.1590/S0034-8910.2014048004822

31. Drumond EF, Souza HNF, Hang-Costa TA. Homicides, alcohol and drugs in Belo Horizonte, Minas Gerais State, Brazil, 2000-2009. Epidemiol Serv Saúde. 2015;24(4):607-16. http://dx.doi.org/10.5123/S1679-49742015000400003

32. Gonzalez-Perez GJ, Vega-López MG, Cabrera-Pivaral CE, VegaLópez A, Munõz la Torre AM. Mortalidad por homicídios en México: tendencias, variaciones socio-geográficas y factores asociados. Cienc Saude Coletiva. 2012;17(12):3195-208 http://dx.doi.org/10.1590/S1413-81232012001200005

33. Dayrell M, Caiaffa WT. Homicídios e consumo de drogas: breve revisão contextualizada em uma zona urbana metropolitana. Rev Med Minas Gerais. 2012;22(3):321-7

34. Singulane BAR, Silva NB, Sartes LMA. Histórico e fatores associados à criminalidade e violência entre dependentes de crack. Psico-USF. 2016;21(2):395-407 http://dx.doi.org/10.1590/1413-82712016210215

35. Reichenheim ME, Souza ER, Moraes CL, Jorge MHM Silva CM, Minayo MCS. Violence and injuries in Brazil: the effect, progress made, and challenges ahead. Lancet. 2011;377(9781):1962-75

http://dx.doi.org/10.1016/S0140-6736(11)60053-6

36. Madruga CS, Laranjeira R, Caetano R, Ribeiro W, Zaleski M, Pinsky I, et al. Early life exposure to violence and substance misuse in adulthood: the first Brazilian national survey. Addict Behav. 2011;36(3):251-5

http://dx.doi.org/10.1016/j.addbeh.2010.10.011

37. Justus M, Kahn T, Cerqueira D. O "Mistério de São Paulo" e o papel do PCC na redução de homicídios nos anos 2000. Instituto de Economia UNICAMP; 2016

38. Brasil. Instituto Brasileiro de Geografia e Estatística (IBGE) Síntese dos indicadores sociais. Uma análise das condições de vida da população brasileira: 2018. Rio de Janeiro: OBGE, 2018.

39. OXFAM Brasil. Um retrato das desigualdades brasileiras: país estagnado. Disponível em: https://www.oxfam.org.br/sites/default/ files/arquivos/relatorio desigualdade 2018 pais estagnado digital. pdf. Acesso em: 18 jan 2019

40. Fundo das Nações Unidas para a Infância (UNICEF). Pobreza na infância e na adolescência. Disponível em: https://www.unicef org/brazil/pt/pobreza infancia adolescencia.pdf. Acesso em: 18 jan 2019. 